\title{
MODELAGEM DA PREVISÃO DE GERAÇÃO DE ENERGIA SOLAR E ANÁLISE ECONÔMICA DA IMPLANTAÇÃO DE PAINÉIS FOTOVOLTAICOS NO IFNMG CAMPUS TEÓFILO OTONI
}

\author{
MODELING AND FORECASTING OF SOLAR ENERGY AND ECONOMIC ANALYSIS OF THE \\ IMPLEMENTATION OF PHOTOVOLTAIC PANELS AT THE IFNMG CAMPUS TEÓFILO OTONI

\section{Larissa Guimarães Rocha ${ }^{1}$, Marcos Oliveira Medeiros ${ }^{2}$, \& Luan Diego de Lima Pereira $^{3 *}$} \\ 123 Grupo de Pesquisa em Energias Renováveis e Eficiência Energética, Instituto Federal do Norte de Minas \\ Gerais, Campus Teófilo Otoni. \\ ${ }^{1}$ lgr2@aluno.ifnmg.edu.br ${ }^{2}$ mmarcos787@gmail.com ${ }^{3 *}$ luan.pereira@ifnmg.edu.br
}

\section{ARTIGO INFO.}

Recebido em: 20.03.2021

Aprovado em: 05.05.2021

Disponibilizado em: 28.05.2021

Palavras-chave:

Previsão de Geração; Correlação; Geração Distribuída; Energia Fotovoltaica.

\section{KEYWORDS:}

Generation Forecast; Correlation; Distributed Generation; Photovoltaic Energy.

*Autor Correspondente: Pereira, L. D. de L.

\section{RESUMO}

Considerando-se o cenário otimista da geração de energia solar no Brasil, o presente artigo apresenta uma modelagem da previsão de geração de energia solar de uma usina solar implantada no Instituto Federal do Norte de Minas Gerais (IFNMG) campus Teófilo Otoni - MG. A metodologia utilizada requer a análise de dados históricos da irradiação solar e temperatura da região de estudo e a utilização da Simulação de Monte Carlo para determinar a previsão de geração de energia. A fim de minimizar o erro causado em utilizar dados médios das variáveis estocásticas, foi feita a modelagem da dependência estocástica entre a temperatura e a irradiação solar. A partir dessa modelagem foi feita uma análise econômica do consumo do IFNMG campus Teófilo Otoni, sendo possível prever a economia gerada pelo sistema de geração distribuída.

\begin{abstract}
Considering the optimistic scenario of the generation of solar energy in Brazil, this paper presents a modeling of the generation forecast of solar energy of a solar power plant implanted in the Federal Institute of the North of Minas Gerais (IFNMG) campus Teófilo Otoni - MG. The methodology used requires the analysis of historical data of the solar irradiation and temperature of the study region and the use of Monte Carlo Simulation to determine the energy generation forecast. In order to minimize the error caused in using average data of the stochastic variables, modeling of the stochastic dependence between temperature and solar irradiation was performed. Based on this modeling, an economic analysis of the consumption of the IFNMG campus Teófilo Otoni was made, making it possible to forecast the savings generated by the distributed generation system.
\end{abstract}


Citação (APA): Rocha, L. G., Medeiros, M. O., \& Pereira, L. D. de L. (2021). Modelagem da previsão de geração de energia solar e análise econômica da implantação de painéis fotovoltaicos no IFNMG campus Teófilo Otoni. Brazilian Journal of Production Engineering, 7(2),7084.

\section{INTRODUÇÃO}

O Brasil apresenta um potencial solar elevado devido às altas incidências de radiação solar em sua região. Devido a este fato, em 2012 entrou em vigor a Resolução Normativa ANEEL n ${ }^{\circ}$ 482/2012, autorizando o consumidor brasileiro a gerar sua própria energia elétrica a partir de fontes renováveis ou cogeração qualificada e inclusive fornecer o excedente para a rede de distribuição de sua localidade (Agência Nacional de Energia Elétrica [ANEEL], 2018).

A Geração Distribuída (GD), tecnologia que gera eletricidade próximo ao local de consumo, apresenta diversas vantagens: proximidade ao consumidor final, reduzindo as perdas com o transporte de energia; menores custos de implantação e rapidez de instalação; e baixo impacto ambiental (Souza, 2014).

Hoje o Brasil apresenta mais de 400.000 sistemas de geração distribuída solar, com uma potência instalada de 4.905,8 MW, onde 18,1\% dessa potência está instalada no estado de Minas Gerais, líder do ranking estadual (Associação Brasileira de Energia Solar Fotovoltaica [ABSOLAR], 2021).

É difícil prever, de forma exata, a geração de energia fotovoltaica, pois ela depende de fatores meteorológicos incertos e ingovernáveis, como a irradiação solar e a temperatura. Por isso existem diversas metodologias que foram desenvolvidas a fim de prever o recurso solar (Domingos et al., 2020).

Fiorotti (2015) propõe em seu trabalho uma metodologia para determinar a potência firme das unidades de geração distribuída aplicando o método de Monte Carlo, capaz de preservar as características estocásticas da potência gerada pelos painéis fotovoltaicos. Segundo ele, este método também serve como uma ferramenta de análise econômica para clientes que possuem tarifas binômia (tarifas de energia e demanda separadas).

Almeida et al. (2017) analisou a viabilidade econômica de uma usina fotovoltaica utilizando dados médios de irradiação solar e considerando a perda de desempenho anual dos módulos solares implantados. Neste trabalho foi discutido a dificuldade da análise econômica devido a volatilidade dos custos de investimento em sistema solar fotovoltaico.

Para esta pesquisa, adotou-se a metodologia probabilística de modelagem de dados respaldados no método de Monte Carlo, através das funções de densidade de probabilidade de séries históricas de irradiação solar e temperatura do município de Teófilo Otoni, em Minas Gerais. De forma a minimizar o erro ao trabalhar com dados médios das variáveis estocásticas, foi feita a modelagem da dependência estocástica entre a temperatura e a irradiação solar.

Além disso, este trabalho realiza uma análise econômica na conta de energia do Instituto Federal do Norte de Minas Gerais (IFNMG) campus Teófilo Otoni, localizado no Vale do Mucuri em Minas Gerais, antes e depois da instalação de duas usinas solares.

\section{Metodologia}

Para este estudo foram coletados dados horários de temperatura e irradiação solar da região de Teófilo Otoni, dos anos de 2014 a 2019, fornecidos pelo Instituto Nacional de Meteorologia

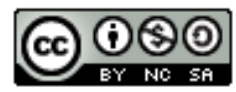


Citação (APA): Rocha, L. G., Medeiros, M. O., \& Pereira, L. D. de L. (2021). Modelagem da previsão de geração de energia solar e análise econômica da implantação de painéis fotovoltaicos no IFNMG campus Teófilo Otoni. Brazilian Journal of Production Engineering, 7(2),7084.

(INMET). Devido à característica de sazonalidade destas grandezas, os dados foram organizados por estações, totalizando 56 intervalos horários (14 horas diárias de irradiação solar x 4 estações).

A previsão de energia foi modelada utilizando-se o Método de Monte Carlo. Essa metodologia se mostra eficaz ao lidar com a característica estocástica da energia solar e é amplamente utilizada na literatura, como em Moradi e Abedini (2012), Oliveira (2013), Ahmadigorji e Amjady (2014), Devi e Geethanjali (2014), Kaur, Kumbhar e Sharma (2014), Fiorotti (2015) e Pereira (2018).

Para a modelagem da irradiação solar foi utilizada a distribuição Beta, pois é a que melhor se adequa quando trabalhamos com dados de irradiação solar. A função de distribuição cumulativa (FDC) de Beta é representada por (1).

$$
f(r)=\frac{\Gamma(\alpha(i)+\beta(i))}{\Gamma(\alpha(i)) \times \Gamma(\beta(i))}\left(\frac{r}{r_{\max }(i)}\right)^{\alpha-1}\left(1-\frac{r}{r_{\max }(i)}\right)^{\beta-1}, \alpha>0, \beta>0
$$

Onde $r$ é a irradiação solar $\left(\mathrm{kW} / \mathrm{m}^{2}\right), r_{\text {max }}(i)$ a irradiação máxima no período i $\left(\mathrm{kW} / \mathrm{m}^{2}\right), \Gamma$ a função gama e $\alpha(i)$ e $\beta(i)$ os parâmetros de forma no período $i$.

Os fatores de forma $\alpha$ e $\beta$ podem ser calculados através da média $\mu$ e desvio padrão $\sigma$ da irradiação solar, como mostrado em (2) e (3). Logo após, é feita a Simulação de Monte Carlo, que atribuiu 10.000 valores aleatórios de irradiação solar a cada um dos 56 intervalos horários. Valores esses que são retirados aleatoriamente da FDC Beta de cada intervalo de tempo (Atwa et al., 2010).

$$
\begin{gathered}
\beta(i)=(1-\mu(i)) \times\left[\left(\frac{\mu(i)(1-\mu(i))}{\sigma(i)^{2}}\right)-1\right],(1 \leq i \leq 56) \\
\alpha(i)=\frac{\mu(i) \beta(i)}{1-\mu(i)},(1 \leq i \leq 56)
\end{gathered}
$$

A FDC da temperatura foi modelada através da distribuição normal, representada por (4).

$$
f(T)=\frac{1}{\sqrt{2 \pi \sigma^{2}}} \exp \left\{-\frac{(T-\mu)^{2}}{2 \sigma^{2}}\right\}
$$

Determinadas as FDCs, a correlação entre a temperatura e a irradiação solar foi calculada conforme a modelagem sugerida por (Othman et al., 2015).

Visando minimizar o erro inerente ao uso de variáveis aleatórias foi utilizado o ranque de correlação $\left(\rho_{r}\right)$. O ranque de correlação das variáveis aleatórias $X$ e $Y$ com FDCs $F_{X}$ e $F_{Y}$ é dado por (5).

$$
\rho_{r}(X, Y)=\rho\left(F_{X}(X), F_{Y}(Y)\right)
$$

Onde $\rho\left(F_{X}(X), F_{Y}(Y)\right)$ é a correlação de Pearson entre as duas FDCs e pode ser calculado por (6).

$$
\rho\left(F_{X}(X), F_{Y}(Y)\right)=\frac{\operatorname{Cov}\left(F_{X}, F_{Y}\right)}{\sigma\left(F_{X}\right) \sigma\left(F_{Y}\right)}
$$

Onde $\operatorname{Cov}$ e $\sigma$ são a covariância e o desvio padrão, respectivamente. O ranque de correlação é simétrico e assume valores no intervalo $[-1,1]$. Este valor indica o grau de relação entre duas 
Citação (APA): Rocha, L. G., Medeiros, M. O., \& Pereira, L. D. de L. (2021). Modelagem da previsão de geração de energia solar e análise econômica da implantação de painéis fotovoltaicos no IFNMG campus Teófilo Otoni. Brazilian Journal of Production Engineering, 7(2),7084.

variáveis quantitativas, no qual, ao se aproximar dos extremos 1 e -1 , significa que há uma relação linear positiva e negativa, respectivamente.

Para variáveis uniformes, a correlação de Pearson é igual ao ranque de correlação $\left(\rho=\rho_{r}\right)$, porém, na maioria dos casos eles não são. A relação entre as duas correlações para distribuições normais multivariadas é dada por (7).

$$
\rho(X, Y)=2 \operatorname{sen}\left(\frac{\pi}{6} \rho_{r}(X, Y)\right)
$$

Onde $X$ e $Y$ são vetores aleatórios com distribuição normal multivariada.

A fim de investigar as propriedades dessas distribuições, é inserida a noção de cópula para a modelagem da dependência estocástica na análise de incerteza. A cópula tem como função separar o efeito da dependência do efeito das distribuições marginais em uma distribuição conjunta, formulando distribuições multivariadas para que qualquer dependência possa ser representada. Cópulas são funções de distribuição multivariada, nas quais as distribuições marginais são uniformes no intervalo $[0,1]$. Duas variáveis aleatórias $X$ e $Y$ podem ser conjuntas pela cópula $\mathrm{C}$ se sua distribuição conjunta puder ser escrita por (8).

$$
F_{X Y}(X, Y)=C\left(F_{X}(X), F_{Y}(Y)\right)
$$

Por definição, se as FDCs são inversíveis, então elas podem ser escritas na forma $F_{X}(X)=u$ e $F_{Y}(Y)=v$, de acordo com a relação (9).

$$
X=F_{X}^{-1}(U) \Leftrightarrow U=F_{X}(X)
$$

Onde $u$ e $v$ são realizações das variáveis aleatórias uniformes $U$ e $V$, respectivamente. Neste caso, (8) torna-se (10).

$$
C_{V \mid U}(u, v)=F(x, y)=F\left(F_{x}^{-1}(u), F_{y}^{-1}(v)\right)
$$

Onde $C_{V \mid U}$ é a distribuição condicional de $V \mid U$ (cópula de $X$ e $Y$ ) e $F^{-1}$ é o inverso da função de distribuição normal univariada padrão.

Para simular a correlação entre as variáveis aleatórias será aplicada a cópula de banda diagonal (CBD), pois ela é facilmente calculada e dispensa aproximações numéricas. Sua representação é dada pela Figura 1. A CBD possui uma largura de banda vertical de $1-\theta, \operatorname{com} \theta \in[0,1]$.

Figura 1. Cópula de banda diagonal.

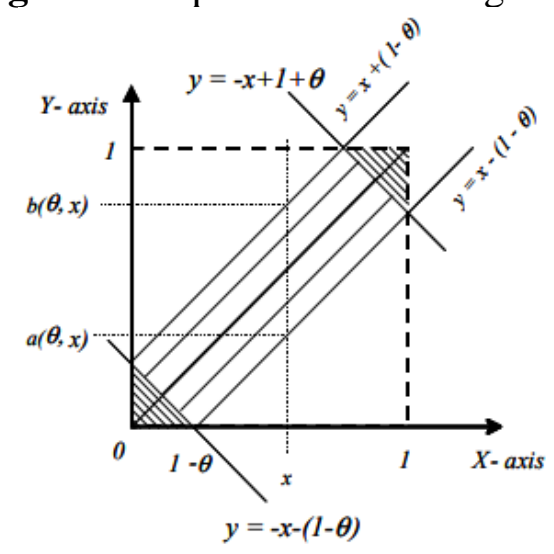

Fonte: Kotz e Dorp (2010).

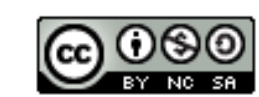


Citação (APA): Rocha, L. G., Medeiros, M. O., \& Pereira, L. D. de L. (2021). Modelagem da previsão de geração de energia solar e análise econômica da implantação de painéis fotovoltaicos no IFNMG campus Teófilo Otoni. Brazilian Journal of Production Engineering, 7(2),7084.

A relação de $\theta$ com o coeficiente de Pearson $(\rho)$ é dada por (11).

$$
\theta=\frac{1}{3}+\frac{4}{3} \operatorname{sen}\left(\frac{1}{3} \operatorname{arcsen}\left(\frac{27}{16}|\rho|-\frac{11}{16}\right)\right)
$$

Com a CBD é possível calcular a distribuição condicional inversa, dada por (12).

$$
C_{V \mid U}^{-1}(t \mid u, \theta)= \begin{cases}(1-\theta) t, & u>1-\theta \text { e } t<1-\frac{u}{1-\theta} \\ (1-\theta) t+\theta, & u>\theta \text { e } t>\frac{1-u}{1-\theta} \\ 2(1-\theta) t+u-1+\theta, & \text { outros }\end{cases}
$$

Para correlacionar a temperatura com a irradiação solar utilizando a CBD, os seguintes passos foram seguidos e iterados para cada hora dos 56 intervalos:

1) Inicia-se com a primeira hora dos 56 intervalos de hora;

2) Gera-se dois vetores independentes $\left(U_{1}\right.$ e $\left.U_{2}\right)$, cada um com 10.000 valores aleatórios uniformemente distribuídos no intervalo $[0,1]$. Denota-se suas realizações por $u_{1}$ e $u_{2}$;

3) Calcula-se o ranque de correlação entre as FDCs Normal $\left(F_{X}(X)\right)$ e Beta $\left(F_{Y}(Y)\right)$ através de (5);

4) Calcula-se o coeficiente de Pearson da temperatura e irradiação solar utilizando (7);

5) Calcula-se $\theta$ para a correlação entre as FDCs Normal e Beta através de (11);

6) Realiza-se a simulação da correlação das variáveis aleatórias com a CBD por meio de (13), onde $C_{V \mid U}^{-1}$ é obtido por (12);

$$
\begin{gathered}
u=u_{1} \\
v=C_{V \mid U}^{-1}\left(u_{2} \mid u, \quad \theta\right)
\end{gathered}
$$

7) Obtém-se as funções de densidade de probabilidade (FDPs) originais das grandezas de temperatura e irradiação solar através da inversa da FDC em (5).

A potência de saída do módulo depende da irradiação solar, da temperatura do ambiente e de características do próprio módulo. Logo, com as FDCs Beta geradas para cada intervalo horário, é calculada a potência de saída simulada dos módulos utilizando as equações de (14) a (18) (Abdelaziz et al., 2014).

$$
\begin{gathered}
T_{c}=T_{a}+r\left(\frac{N_{o t}-20}{0,8}\right) \\
I=r\left[I_{s c}+K_{i}\left(T_{c}-25\right)\right] \\
V=V_{o c}-K_{v} \times T_{c} \\
F F=\frac{V_{m p p} \times I_{m p p}}{V_{o c} \times I_{s c}} \\
P_{S}=F F \times V \times I
\end{gathered}
$$

Em que $T_{c}$ é a temperatura do painel em ${ }^{\circ} \mathrm{C} ; T_{a}$ a temperatura média do ambiente em ${ }^{\circ} \mathrm{C} ; N_{o t}$ a temperatura nominal de operação da célula em ${ }^{\circ} \mathrm{C} ; I$ a corrente na célula (A); $I_{s c}$ a corrente de curto circuito (A); $K_{i}$ é o coeficiente corrente/temperatura $\left(\mathrm{A} /{ }^{\circ} \mathrm{C}\right) ; V$ a tensão do módulo fotovoltaico $(\mathrm{V}) ; V_{o c}$ a tensão de circuito aberto do módulo (V); $K_{v}$ o coeficiente

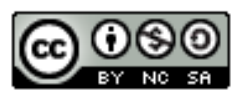


Citação (APA): Rocha, L. G., Medeiros, M. O., \& Pereira, L. D. de L. (2021). Modelagem da previsão de geração de energia solar e análise econômica da implantação de painéis fotovoltaicos no IFNMG campus Teófilo Otoni. Brazilian Journal of Production Engineering, 7(2),7084.

tensão/temperatura $\left(\mathrm{V} /{ }^{\circ} \mathrm{C}\right) ; F F$ o fator de forma; $V_{m p p}$ a tensão no ponto de máxima potência $(\mathrm{V}) ; I_{m p p}$ a corrente no ponto de máxima potência (A); $P_{S}$ a potência de saída simulada do painel (Tabela 1).

Tabela 1. Especificações técnicas dos painéis fotovoltaicos utilizados.

\begin{tabular}{lcc}
\hline Especificações técnicas & Usina 1 (Canadian Solar) & Usina 2 (Astronergy Solar) \\
\hline Potência de pico (Wp) & 405,00 & 345,00 \\
Tensão em circuito aberto (V) & 47,80 & 46,37 \\
Corrente de curto-circuito (A) & 11,14 & 9,67 \\
Tensão no ponto de máxima potência (V) & 39,30 & 37,38 \\
Corrente no ponto de máxima potência (A) & 10,56 & 9,23 \\
Coeficiente de tensão/temperatura (V/ $\left./{ }^{\circ} \mathrm{C}\right)$ & 0,29 & 0,311 \\
Coeficiente de corrente/temperatura $\left(\mathrm{A} /{ }^{\circ} \mathrm{C}\right)$ & 0,05 & 0,05 \\
Temperatura nominal de operação da célula $\left({ }^{\circ} \mathrm{C}\right)$ & 41,00 & 46,00 \\
\hline
\end{tabular}

Fonte: Adaptado de Canadian Solar (2020); Astronergy Solar (2018).

Obtida a previsão de geração da GD em questão foi então possível calcular os custos com energia elétrica. De posse dos registros de consumo e demanda do IFNMG campus Teófilo Otoni no ano de 2019 foi possível realizar as análises econômicas após a implantação da GD.

$\mathrm{Na}$ fatura de energia são cobradas a energia consumida, o uso do sistema de distribuição, a demanda contratada e os tributos federais (PIS e COFINS), estaduais (ICMS) e municipais (taxa de iluminação pública). A tarifa de energia (TE) é relativa ao custo de energia consumida em kWh; a tarifa de uso do Sistema de Distribuição (TUSD) é a referente a custos na transmissão e distribuição de energia; e a tarifa de demanda é cobrada por kW disponível na entrada do estabelecimento do consumidor. A demanda é cobrada de acordo com o valor contratado pela unidade consumidora, independente se foi consumida ou não e, caso exceda o valor de contrato, pode ocorrer taxação extra (ANEEL, 2010).

Para o grupo A, modalidade tarifária horária verde, há distinção horária para consumo de energia elétrica, possuindo tarifas diferentes para horário de ponta e fora de ponta. O horário de ponta da região do estudo está compreendido entre 17 e 20 horas.

O cálculo do gasto de energia ativa (GEA) e do gasto com energia reativa excedente (GERE), em reais, é realizado por (19) e (20), nas quais os sufixos P e FP representam horário de ponta e fora de ponta, respectivamente. A energia reativa excedente (ERE), em $\mathrm{kWh}$, equivale ao produto do montante de energia elétrica ativa medida pelo percentual do fator de potência excedente, estabelecido pela ANEEL em 0,92.

$$
\begin{gathered}
G E A=E_{P} \times\left(T E_{P}+T U S D_{P}\right)+E_{F P} \times\left(T E_{F P}+T U S D_{F P}\right) \\
G E R E=\left(E R E_{P}+E R E_{F P}\right) \times T_{R}
\end{gathered}
$$

Onde $E$ é a energia consumida $(\mathrm{kWh})$ e $T_{R}$ é o valor de referência equivalente à TE da bandeira verde aplicável ao subgrupo B1 (R\$/MWh). 
Citação (APA): Rocha, L. G., Medeiros, M. O., \& Pereira, L. D. de L. (2021). Modelagem da previsão de geração de energia solar e análise econômica da implantação de painéis fotovoltaicos no IFNMG campus Teófilo Otoni. Brazilian Journal of Production Engineering, 7(2),7084.

Para o gasto com demanda de potência, o cálculo é realizado em função da demanda medida, havendo três possibilidades: menor que a contratada, entre $100 \%$ e $105 \%$ da contratada e maior que $105 \%$ da contratada, conforme (21), (22) e (23), respectivamente.

$$
\begin{gathered}
G_{D}=D_{c} \times T U S D \\
G_{D}=D_{m} \times T U S D \\
G_{D}=\left(D_{m}+2 \times\left(D_{m}-D_{c}\right)\right) \times T U S D
\end{gathered}
$$

Onde $D_{m}$ é a demanda medida $(\mathrm{kW})$ e $D_{c}$ é a demanda contratada $(\mathrm{kW})$.

As tarifas utilizadas neste estudo estão de acordo com a Resolução Homologatória ${ }^{\circ}$ 2.757, de 18 de agosto de 2020 .

\section{Resultados e Discussão}

Para este estudo foi considerada a microgeração distribuída implantada do IFNMG campus Teófilo Otoni, composta por duas usinas distintas de geração solar, uma com 46 painéis fotovoltaicos e outra com 162 painéis fotovoltaicos.

Os dados históricos da irradiação solar e da temperatura da região de Teófilo Otoni - MG, foram organizados em 56 intervalos horários, correspondentes às 14 horas com incidência solar das quatro estações do ano, sendo representados seus valores médios nas Figuras 2 e 3, respectivamente.

Figura 2. Série histórica da irradiação solar em 56 intervalos horários.

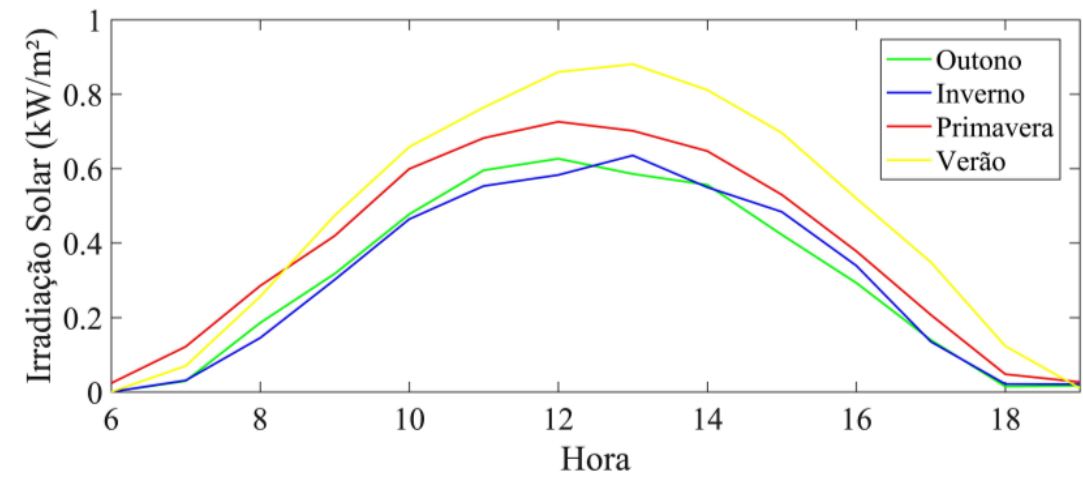

Fonte: Autores (2021).

Figura 3. Valores médios de temperatura para os 56 intervalos horários.

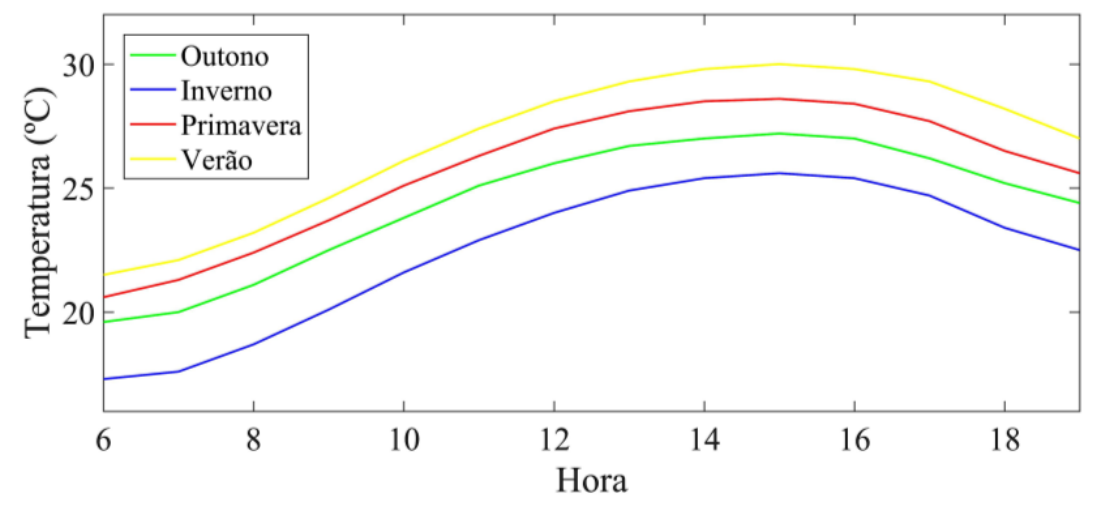

Fonte: Autores (2021). 
Citação (APA): Rocha, L. G., Medeiros, M. O., \& Pereira, L. D. de L. (2021). Modelagem da previsão de geração de energia solar e análise econômica da implantação de painéis fotovoltaicos no IFNMG campus Teófilo Otoni. Brazilian Journal of Production Engineering, 7(2),7084.

A Figura 4 mostra o ranque de correlação entre a temperatura e a irradiação solar para os 56 intervalos horários. Pode-se perceber que há uma correlação, de moderada a forte, entre a temperatura e a irradiação solar e, quanto mais alta a irradiação solar (próximo às 12h), mais forte a dependência com a temperatura.

Figura 4. Coeficiente de Pearson entre a temperatura e irradiação solar para os 56 intervalos horários.

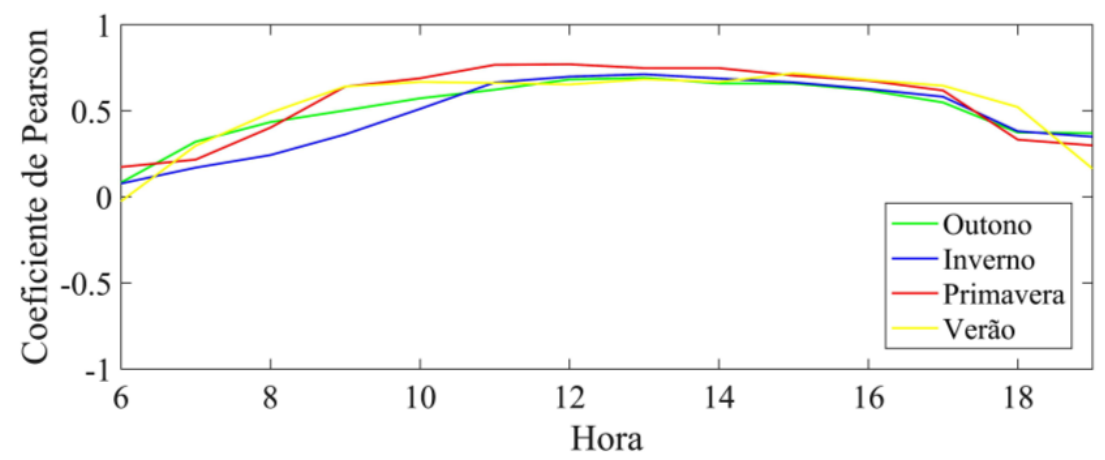

Fonte: Autores (2021).

A potência gerada pela GD foi simulada inicialmente desconsiderando a dependência estocástica entre a temperatura e a irradiação solar. Nesta primeira análise foram utilizados os dados médios da irradiação solar e da temperatura, que podem ser observados nas Figuras 2 e 3 , respectivamente. Os valores de energia gerada por mês e por estação estão apresentados nas Figuras 5 e 6, respectivamente.

Figura 5. Geração anual de energia.

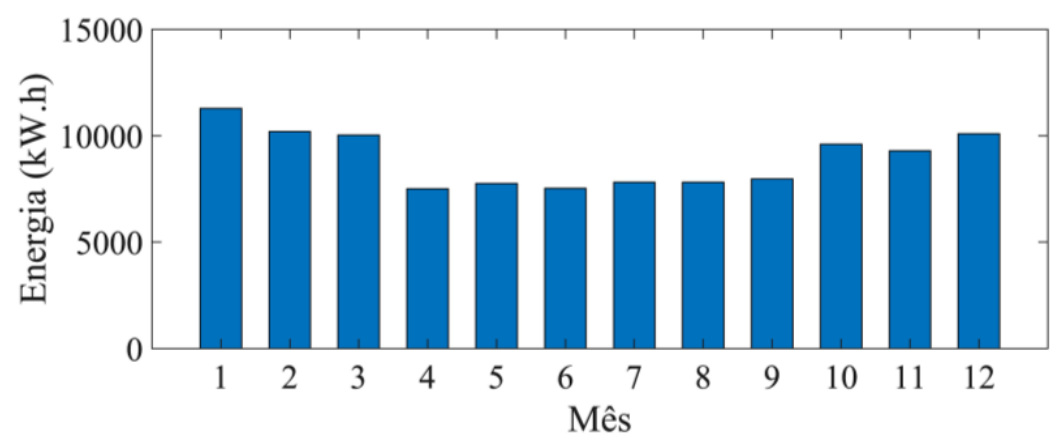

Fonte: Autores (2021).

Figura 6. Geração de energia por estação.

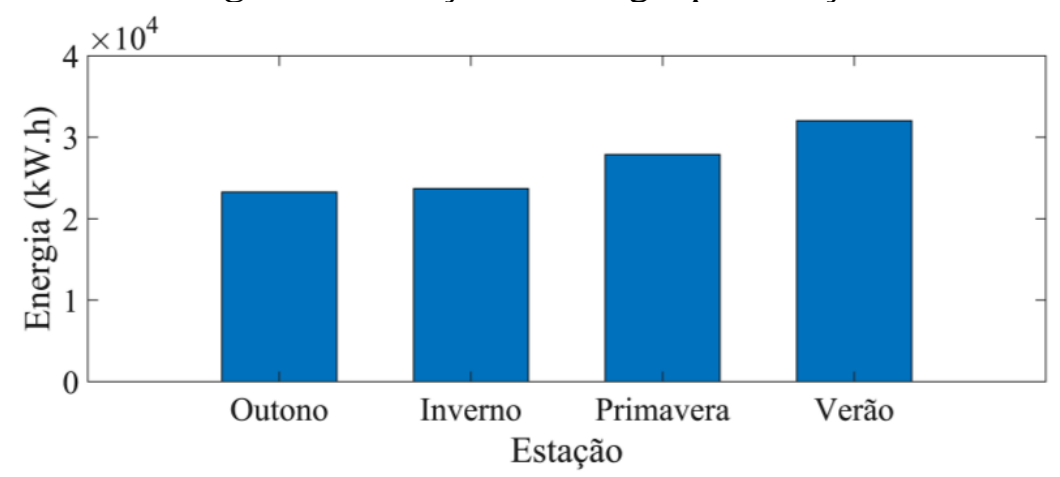

Fonte: Autores (2021).

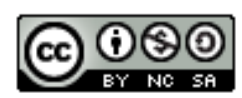


Citação (APA): Rocha, L. G., Medeiros, M. O., \& Pereira, L. D. de L. (2021). Modelagem da previsão de geração de energia solar e análise econômica da implantação de painéis fotovoltaicos no IFNMG campus Teófilo Otoni. Brazilian Journal of Production Engineering, 7(2),7084.

Observa-se que há geração durante todo o ano, e que ela tende a ser maior nos meses que compreendem o verão, sendo janeiro o mês com maior geração de energia.

Agora, foi considerada a dependência estocástica entre a temperatura e a irradiação solar para a modelagem da potência gerada. Neste caso, utilizamos os dados de temperatura e irradiação solar obtidos da aplicação da CBD, apresentados nas Figuras 7 e 8, respectivamente.

Observando as Figuras 3 e 7, pode-se notar que não teve alteração nos valores da temperatura, pois a temperatura é a variável independente da correlação. Já a irradiação solar segue o mesmo padrão, porém apresenta valores menores (Figura 8), efeito da dependência estocástica moderada.

Figura 7. Valores de temperatura obtidos da modelagem de dependência estocástica para os 56 intervalos horários.

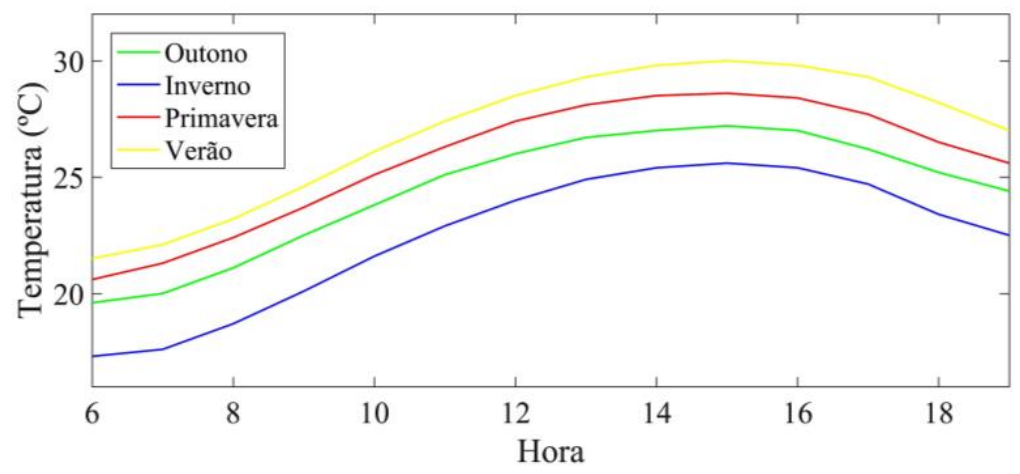

Fonte: Autores (2021).

Figura 8. Valores de irradiação solar obtidos da modelagem de dependência estocástica para os 56 intervalos horários.

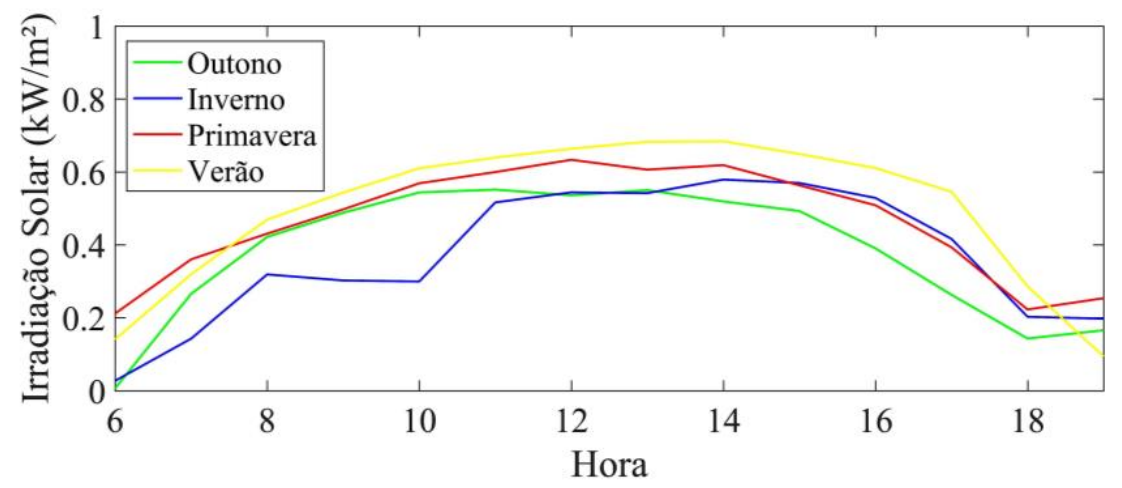

Fonte: Autores (2021).

Nas Figuras 9 e 10 estão apresentados os valores de geração de energia por mês e por estação, respectivamente. Pode-se observar agora que, após a modelagem da dependência estocástica entre a temperatura e a irradiação solar, houve alterações de valores, tornando a previsão mais confiável. Com valores médios, a geração de energia anual foi de 106.910,00 kWh, enquanto a geração com dados correlacionados foi de 125.130,00 kWh, representando um aumento de $17 \%$, aproximadamente. 
Citação (APA): Rocha, L. G., Medeiros, M. O., \& Pereira, L. D. de L. (2021). Modelagem da previsão de geração de energia solar e análise econômica da implantação de painéis fotovoltaicos no IFNMG campus Teófilo Otoni. Brazilian Journal of Production Engineering, 7(2),7084.

Figura 9. Geração anual de energia da GD considerando a dependência estocástica entre a temperatura e irradiação solar.

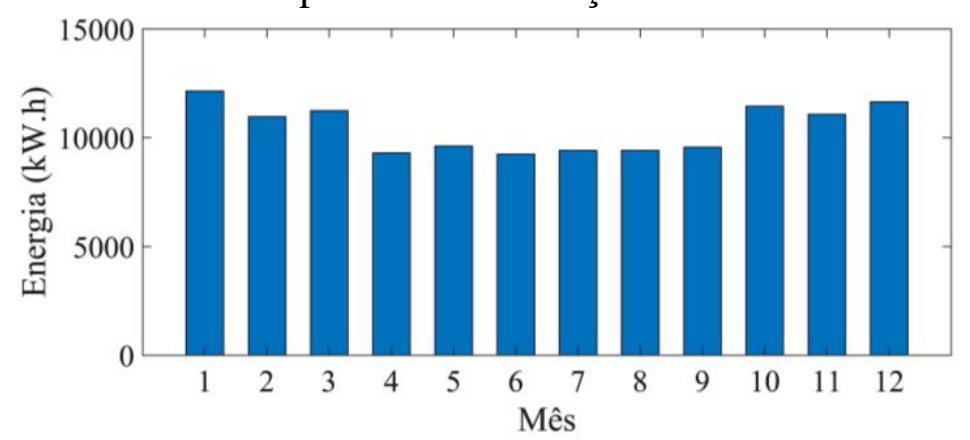

Fonte: Autores (2021).

Figura 10. Geração de energia da GD por estação considerando a dependência estocástica

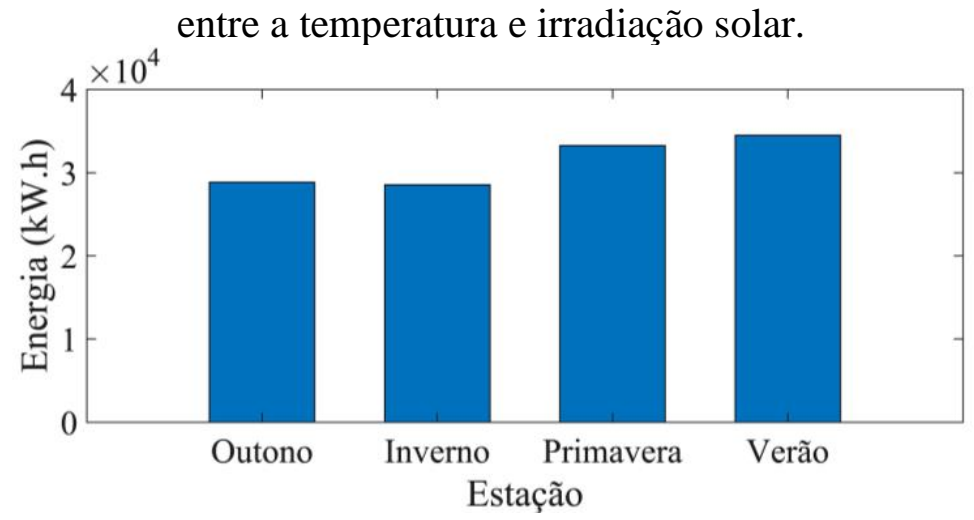

Fonte: Autores (2021).

Para analisar o impacto das GD na conta de energia do IFNMG campus Teófilo Otoni, foram estimados os valores gastos com consumo e demanda de potência. A economia gerada pela parcela de energia é calculada subtraindo a energia consumida pelo instituto da gerada pelas GD, no mesmo posto horário. A parcela da demanda é a diferença entre potência ativa consumida e potência ativa gerada dentro do mesmo posto horário. A Tabela 2 mostra o consumo do ano de 2019 e geração de energia prevista nos horários de ponta (HP) e fora de ponta (HFP).

Tabela 2. Consumo e geração de energia.

\begin{tabular}{lccccc}
\hline Mês & $\begin{array}{c}\text { Consumo HP } \\
(\mathbf{k W h})\end{array}$ & $\begin{array}{c}\text { Geração HP } \\
(\mathbf{k W h})\end{array}$ & $\begin{array}{c}\text { Consumo HFP } \\
(\mathbf{k W h})\end{array}$ & $\begin{array}{c}\text { Geração HFP } \\
(\mathbf{k W h})\end{array}$ & $\begin{array}{c}\text { Crédito } \\
(\mathbf{k W h})\end{array}$ \\
\hline Janeiro & 500,98 & 497,47 & 6390,64 & 11651,90 & 5261,26 \\
Fevereiro & 1611,25 & 452,24 & 9782,64 & 10521,38 & 738,74 \\
Março & 1517,42 & 423,22 & 10303,36 & 10827,35 & 523,99 \\
Abril & 1603,39 & 387,78 & 11249,74 & 8918,39 & 0,00 \\
Maio & 1271,09 & 406,24 & 8429,32 & 9210,13 & 780,82 \\
Junho & 860,75 & 379,39 & 6731,59 & 8868,20 & 2136,61 \\
Julho & 839,72 & 604,35 & 6860,52 & 8810,25 & 1949,73 \\
Agosto & 947,39 & 531,82 & 7113,33 & 8882,77 & 1769,44 \\
Setembro & 942,90 & 527,63 & 7050,46 & 9042,91 & 1992,45 \\
Outubro & 1109,05 & 647,92 & 7718,17 & 10802,25 & 3084,07 \\
Novembro & 1348,96 & 563,41 & 8899,21 & 10517,40 & 1618,19 \\
Dezembro & 878,93 & 558,23 & 6615,25 & 11094,93 & 4479,68 \\
\hline Total & $\mathbf{1 3 4 3 1 , 8 2}$ & $\mathbf{5 9 7 9 , 7 0}$ & $\mathbf{9 7 1 4 4 , 2 2}$ & $\mathbf{1 1 9 1 4 7 , 8 5}$ & $\mathbf{2 4 3 3 4 , 9 8}$ \\
\hline
\end{tabular}

Fonte: Autores (2021).

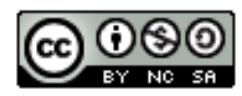


Citação (APA): Rocha, L. G., Medeiros, M. O., \& Pereira, L. D. de L. (2021). Modelagem da previsão de geração de energia solar e análise econômica da implantação de painéis fotovoltaicos no IFNMG campus Teófilo Otoni. Brazilian Journal of Production Engineering, 7(2),7084.

O consumo de energia no horário de ponta foi superior à geração, devido à baixa incidência de irradiação solar do período. Em contrapartida, a geração no horário fora de ponta foi superior ao consumo em quase todos os meses do ano, gerando um acumulo de créditos, que podem ser utilizados posteriormente. A legislação não permite o uso de créditos em posto horário diferente daquele que foi gerado, portanto, não foi possível zerar o consumo faturado de energia da unidade consumidora.

O uso da geração distribuída também gera impactos na demanda e na energia reativa excedente faturadas dos consumidores do tipo A. A Tabela 3 mostra os dados de demandas consumida no ano de 2019, sem utilização de GD, e previstas com o uso de GD, assim como a energia reativa excedente.

Tabela 3. Dados de demanda e energia reativa excedente.

\begin{tabular}{ccccc}
\hline Mês & $\begin{array}{c}\text { Demanda sem GD } \\
(\mathbf{k W})\end{array}$ & $\begin{array}{c}\text { Demanda com GD } \\
(\mathbf{k W})\end{array}$ & $\begin{array}{c}\text { ERE sem GD } \\
(\mathbf{k W})\end{array}$ & $\begin{array}{c}\text { ERE com GD } \\
(\mathbf{k W})\end{array}$ \\
\hline Janeiro & 21,25 & 12,85 & 502,59 & 130,63 \\
Fevereiro & 51,32 & 41,66 & 241,54 & 280,32 \\
Março & 54,56 & 45,49 & 410,18 & 338,21 \\
Abril & 56,83 & 40,07 & 261,57 & 455,11 \\
Maio & 49,94 & 34,15 & 365,57 & 268,76 \\
Junho & 29,61 & 21,63 & 447,17 & 135,37 \\
Julho & 27,03 & 27,03 & 512,02 & 71,90 \\
Agosto & 26,80 & 21,17 & 448,34 & 54,14 \\
Setembro & 28,18 & 22,97 & 417,78 & 61,49 \\
Outubro & 45,40 & 38,39 & 521,26 & 169,94 \\
Novembro & 59,39 & 39,94 & 457,19 & 246,46 \\
Dezembro & 34,31 & 33,10 & 651,29 & 128,10 \\
\hline Total & $\mathbf{4 5 0 , 3 1}$ & $\mathbf{3 7 8 , 4 4}$ & $\mathbf{4 5 8 5 , 2 2}$ & $\mathbf{2 3 4 0 , 4 3}$ \\
\hline
\end{tabular}

Fonte: Autores (2021).

O IFNMG campus Teófilo Otoni possui um contrato de demanda de $94 \mathrm{~kW}$, estando, portanto, mal dimensionado, uma vez que, a maior demanda consumida, sem GD, ocorreu no mês de novembro, com 59,39 kW e, com GD, em março com 45,49 kW. A inserção da GD impactou em uma redução média de 8 a $9 \mathrm{~kW}$ de demanda mensal.

A Figura 11 apresenta o gasto anual com energia elétrica antes e depois da implantação da GD no IFNMG campus Teófilo Otoni. Já a Figura 12 mostra os valores das faturas mensais.

Figura 11. Gasto anual com energia elétrica com e sem GD.
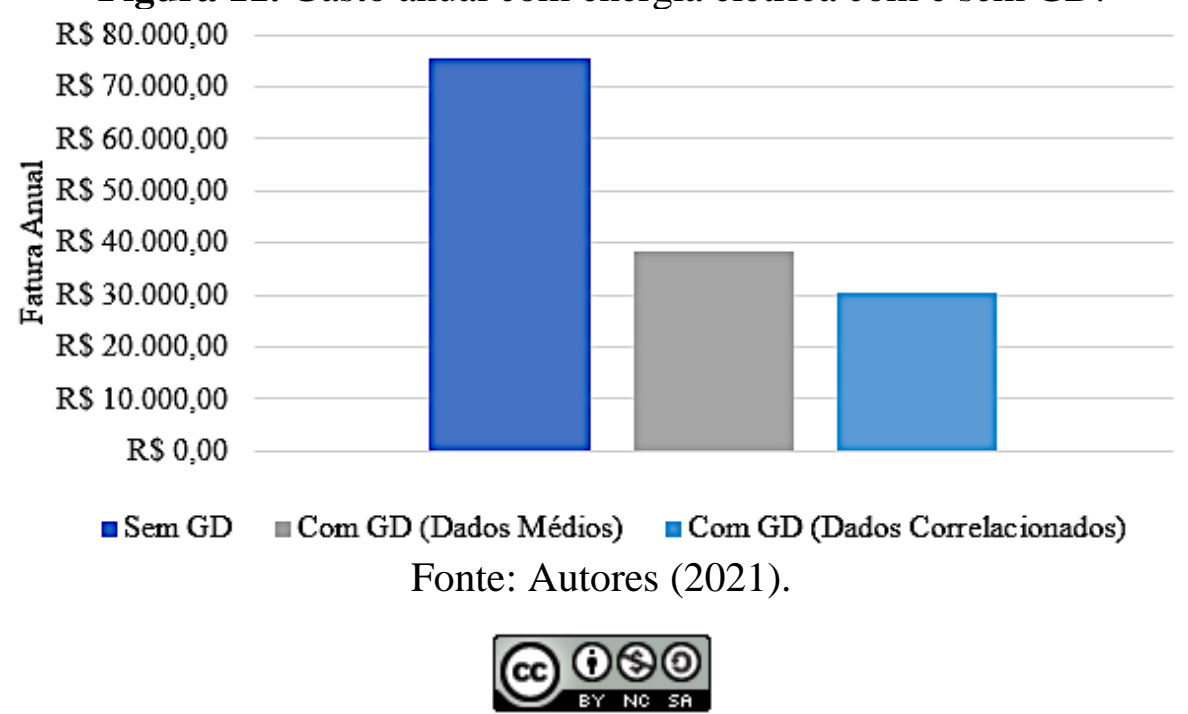
Citação (APA): Rocha, L. G., Medeiros, M. O., \& Pereira, L. D. de L. (2021). Modelagem da previsão de geração de energia solar e análise econômica da implantação de painéis fotovoltaicos no IFNMG campus Teófilo Otoni. Brazilian Journal of Production Engineering, 7(2),7084.

Figura 32. Faturas mensais de energia elétrica com e sem GD.

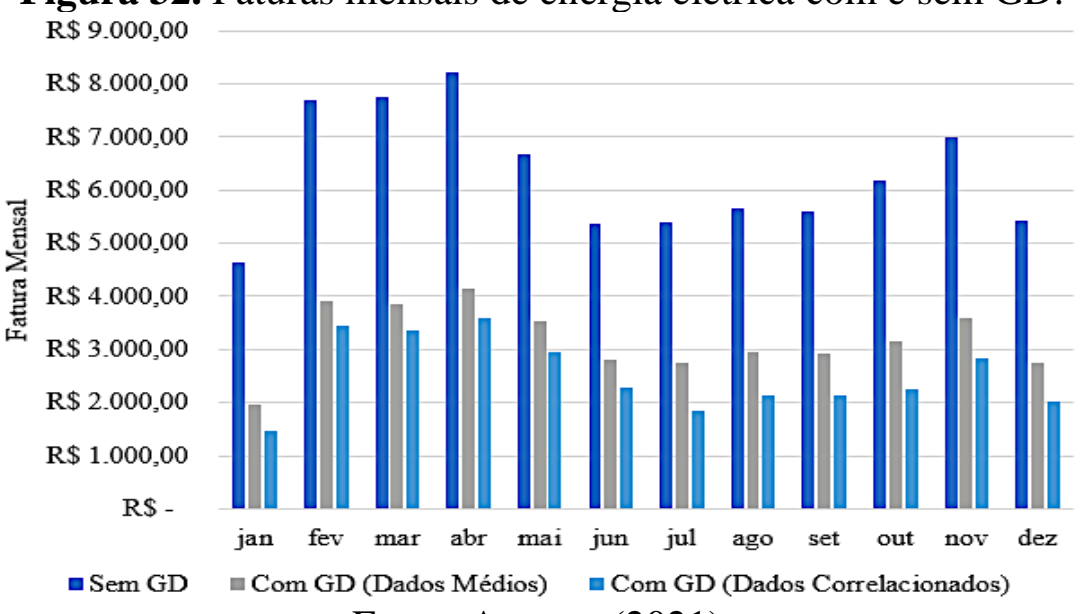

Fonte: Autores (2021).

De acordo com os dados obtidos há uma economia no valor gasto com o consumo total em todos os meses do ano. A estimativa é que haja uma economia anual de R \$37.209,04 com energia, quando considerado os dados médios de temperatura e irradiação solar. A economia é ainda maior quando se leva em consideração a dependência estocástica das variáveis aleatórias, aumentando para $\mathrm{R} \$ 45.185,33$.

A Tabela 4 apresenta o gasto anual da conta de energia do IFNMG campus Teófilo Otoni, subdivido pelas categorias: demandas consumida e não consumida, energia consumida, energia reativa excedente e impostos (ICMS, PASEP e COFINS) e taxa de iluminação pública.

Tabela 4. Fatura anual com e sem GD.

\begin{tabular}{cccc}
\hline Categoria & Sem GD $(\mathbf{R} \mathbf{\text { ) }}$ & Com GD $(\mathbf{R} \mathbf{\$})$ & Economia $(\mathbf{R} \mathbf{\$})$ \\
\hline Demanda consumida & $7.114,22$ & $5.555,56$ & $1.588,66$ \\
Demanda não consumida & $9.444,82$ & $11.003,48$ & $-1.588,66$ \\
Energia consumida & $54.644,09$ & $11.929,21$ & $42.714,88$ \\
ERE & $1.384,27$ & 618,69 & 765,58 \\
Impostos e Iluminação pública & $2.996,55$ & $1.291,68$ & $1.704,87$ \\
\hline Total & $\mathbf{7 5 . 5 8 3 , 9 5}$ & $\mathbf{3 0 . 3 9 8 , 6 2}$ & $\mathbf{4 5 . 1 8 5 , 3 3}$ \\
\hline
\end{tabular}

Fonte: Autores (2021).

Com a inserção da GD na unidade consumidora seria possível ter uma economia total de aproximadamente $60 \%$, decorrentes da redução dos custos com energia consumida, energia reativa excedente, impostos e taxa de iluminação pública. Apesar de ter uma redução de custos de aproximadamente $22 \%$ com a demanda consumida, essa redução não impacta no faturamento, uma vez que, consumidores do tipo A pagam pela demanda contratada, independente se há ou não o consumo total. Para haver redução de fato no faturamento, é preciso revisar o valor de demanda contratada. A Tabela 5 mostra uma simulação de readequação do contrato de demanda atual, sem GD, e com o cenário do uso de GD. Os valores simulados levaram em consideração o maior valor de demanda consumida na unidade consumidora no ano de 2019, evitando, portanto, multas por ultrapassar o valor de contrato.

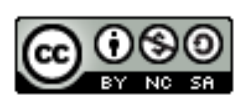


Citação (APA): Rocha, L. G., Medeiros, M. O., \& Pereira, L. D. de L. (2021). Modelagem da previsão de geração de energia solar e análise econômica da implantação de painéis fotovoltaicos no IFNMG campus Teófilo Otoni. Brazilian Journal of Production Engineering, 7(2),7084.

Tabela 5. Simulação de readequação do contrato de demanda.

\begin{tabular}{ccccc}
\hline \multirow{2}{*}{ Mês } & \multicolumn{2}{c}{ Contrato 60 kW (sem GD) } & \multicolumn{2}{c}{ Contrato 46 kW (com GD) } \\
\cline { 2 - 5 } & $\begin{array}{c}\text { Demanda } \\
\text { consumida (R\$) }\end{array}$ & $\begin{array}{c}\text { Demanda não } \\
\text { consumida (R\$) }\end{array}$ & $\begin{array}{c}\text { Demanda } \\
\text { consumida (R\$) }\end{array}$ & $\begin{array}{c}\text { Demanda não } \\
\text { consumida (R\$) }\end{array}$ \\
\hline Janeiro & 188,67 & 692,13 & 188,67 & 486,61 \\
Fevereiro & 611,63 & 269,17 & 611,63 & 63,65 \\
Março & 667,73 & 213,07 & 667,73 & 7,55 \\
Abril & 588,20 & 292,60 & 588,20 & 87,08 \\
Maio & 501,26 & 379,54 & 501,26 & 174,02 \\
Junho & 317,53 & 563,27 & 317,53 & 357,75 \\
Julho & 396,80 & 484,00 & 396,80 & 278,48 \\
Agosto & 310,75 & 570,05 & 310,75 & 364,53 \\
Setembro & 337,26 & 543,54 & 337,26 & 338,02 \\
Outubro & 563,54 & 317,26 & 563,54 & 111,74 \\
Novembro & 586,35 & 294,45 & 586,35 & 88,93 \\
Dezembro & 485,85 & 394,95 & 485,85 & 189,43 \\
\hline Total & $\mathbf{5 . 5 5 5 , 5 6}$ & $\mathbf{5 . 0 1 4 , 0 4}$ & $\mathbf{5 . 5 5 5 , 5 6}$ & $\mathbf{2 . 5 4 7 , 8 0}$ \\
\hline
\end{tabular}

Fonte: Autores (2021).

Comparando os valores anuais de demanda faturada (soma da consumida com a não consumida) das Tabelas 4 e 5, observa-se que, mesmo sem GD, com a readequação da demanda contratada haveria uma redução de $\mathrm{R} \$ 5989,44$ no faturamento anual. Com a inserção da GD a demanda consumida diminui, possibilitando readequar o valor de contrato novamente e obter uma economia de $\mathrm{R} \$ 2466,24$.

\section{CONSIDERAÇÕES FINAIS}

O setor solar fotovoltaico vem crescendo nos últimos anos e boa parte do crescimento é devido a instalações de geração distribuída. Considerando este cenário, foi apresentado neste trabalho uma metodologia para obter o modelo probabilístico da geração de energia elétrica através de painéis fotovoltaicos na região de Teófilo Otoni, município de Minas Gerais.

Ao modelar a correlação entre a temperatura e a irradiação solar, a fim de reduzir o erro gerado ao trabalhar com variáveis aleatórias, observou-se uma dependência de moderada a forte entre a temperatura e a irradiação solar.

Foram apresentados os resultados considerando tanto os valores médios, quanto os correlacionados. Com a correlação entre a irradiação solar e a temperatura foi possível minimizar as incertezas, oriundas de grandezas estocásticas, tornando a previsão da geração mais assertiva.

A partir da análise tarifária de energia elétrica do IFNMG campus Teófilo Otoni, foi possível prever a economia após a implantação de usinas de geração distribuída no campus. A economia prevista supera $\mathrm{R} \$ 45.000,00$, no cenário mais otimista. Além disso, há uma redução no

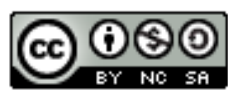


Citação (APA): Rocha, L. G., Medeiros, M. O., \& Pereira, L. D. de L. (2021). Modelagem da previsão de geração de energia solar e análise econômica da implantação de painéis fotovoltaicos no IFNMG campus Teófilo Otoni. Brazilian Journal of Production Engineering, 7(2),7084.

consumo de demanda, possibilitando readequar o valor contratado da concessionária e, consequentemente, aumentar a economia na fatura.

\section{REFERÊNCIAS}

Abdelaziz, A. Y., Hegazy, Y. G., El-Khattam, W., \& Othman, M. M. (2014). Optimal allocation of stochastically dependent renewable energy based distributed generators in unbalanced distribution networks. Electric Power Systems Research. Egito, Cairo.

Agência Nacional de Energia Elétrica (2018). Geração Distribuída. Brasília/DF, 15/08/2018. Recuperado de: <https://www.aneel.gov.br/geracao-distribuida〉. Acesso em: 08/09/2020.

Agência Nacional de Energia Elétrica (2010). Resolução normativa ANEEL. no 414/2010 de 09 de setembro de 2010.20 Recuperado https://www.aneel.gov.br/documents/656877/14486448/bren2010414.pdf/3bd33297-26f94ddf-94c3-f01d76d6f14a?version=1.0

Ahmadigorji, M., \& Amjady, N. A. (2014). new evolutionary solution method for dynamic expansion planning of DG-integrated primary distribution networks. Energy Conversion and Management,82:61-70.

Almeida, R. R. G., Brito, N. S. D., Medeiros, M. V. B., Simões, M. C. S., \& Oliveira, S. A. (2017). Proposição de uma metodologia para análise de viabilidade econômica de uma usina fotovoltaica. Congresso Brasileiro de Educação em Engenharia. João Pessoa.

Associação Brasileira de Energia Solar Fotovoltaica (2021). Agência Nacional de Energia Elétrica. Infográfico ABSOLAR $n^{o}$ 29. São Paulo, 02/03/2021. Recuperado de https://www.absolar.org.br/mercado/infografico/

Astronergy Solar, "STAVE II 330W 345W 5BB-Polycrystalline PV Module", 2018. Recuperado de http://www.astronergy.com/attch/product/STAVE\%20II_CHSM6612P_40mm\%20frame_201 801.pdf

Atwa Y. M., El-Saadany E. F., Salama M. M. A., \& Seethapathy, R. (2010). Optimal renewable resources mix for distribution system energy loss minimization. IEEE Transactions on Power Systems.

Canadian Solar, "Super high power poly perc module $395 \mathrm{~W} \sim 420 \mathrm{~W}$ ", 2020. Recuperado de https://www.canadiansolar.com/wp-content/uploads/2019/12/Canadian_Solar-DatasheetHiKu_CS3W-P_EN.pdf

Devi, S., \& Geethanjali, M. (2014). Optimal location and sizing determination of distributed generation and DSTATCOM using Particle Swarm Optimization algorithm. International Journal of Electrical Power \& Energy Systems, 62:562-570.

Domingos, S. F., Monteiro, L. G., \& Boaventura, W. C. Estado da Arte para Previsão da Radiação Solar. VIII Congresso Brasileiro de Energia Solar. Fortaleza, 2020.

Fiorotti, R. (2015). Metodologia para determinar a potência firme das unidades de geração distribuída e sua aplicação no processo de previsão de demanda das redes de distribuição de energia elétrica (Dissertação de Mestrado). Universidade Federal do Espírito Santo, Vitória.

Kaur, S., Kumbhar, G., \& Sharma, J. A Minlp. (2014). technique for optimal placement of multiple DG units in distribution systems. International Journal of Electrical Power \& Energy Systems, 63:609-617.

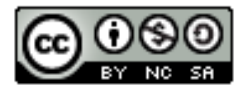


Kotz, S., \& Dorp, J. R. (2010). Generalized diagonal band copulas with two-sided generating densities. Decision Analysis. 196-214.

Moradi, M. H., \& Abedini, M. A. (2012). combination of genetic algorithm and particle swarm optimization for optimal distributed generation location and sizing in distribution systems with Fuzzy optimal theory. International Journal of Green Energy, 9:641-660.

Othman, M. M., Abdelaziz, A. Y., Hegazy, Y. G., \& El-Khattam, W. (2015). Approach for modelling stochastically dependent renewable energy-based generators using diagonal band copula. The Institution of Engineering and Technology Journals. Egito, Cairo.

Pereira, L. D. de L. (2018). Alocação ótima de Geração Distribuída em sistemas de distribuição considerando incertezas no modelo probabilístico de geração (Dissertação de Mestrado). Centro Tecnológico da Universidade Federal do Espírito Santo, Vitória.

Souza, M. E. M. (2014). Inserção de Microgeração Distribuída nas redes de baixa tensão: implantação de telhados solares (Dissertação de Mestrado). Escola de Engenharia, Universidade Federal de Minas Gerais, Minas Gerais. 